\title{
Hierarchical Routing Protocol for Enhancement in Efficiency of Wireless Sensor Nodes
}

\author{
*Nishant Juneja ${ }^{1}$, Barinder Paul Singh ${ }^{2}$ \\ ${ }^{I}$ P.G. Department of Mathematics, Dev Samaj College for Women, Ferozepur, India \\ ${ }^{2}$ Department of ECE, Faculty of Technical Education, Ferozepur College of Engineering and Technology, \\ Ferozeshah, Ferozepur, India \\ Corresponding Author: Nishant Juneja
}

\begin{abstract}
Sensors in most of the Wireless Sensor Networks (WSN) rely on batteries as the source of energy. The power backup is one of the major constraints. It is impractical to recharge or reinstate sensor nodes as they are deployed or located in the areas which are not easily accessible by humans. So Efficient-Energy Coverage (EEC) has become challenge issue for WSN. So the lifetime of a network can be prolonged and energy consumption be minimized by scheduling the actions of these nodes. The present paper deals with an Ant Colony Based Scheduling Algorithm (ACB-SA) to resolve the EEC issue. We apply ACB-SA to a heterogeneous sensor set by the execution of PSD model. The simulation results are carried out by using MATLAB.
\end{abstract}

Keywords: Probabilistic Sensor Detection (PSD), Efficient Energy Coverage (EEC), Ant Colony Optimization (ACO), Ant Colony Based Scheduling Algorithm (ACB-SA), Base Station (BS).

\section{Introduction}

A Wireless Sensor Network (WSN) is composed of vast amount of minute wireless sensor nodes that are densely structured. A sensor network consists of large number of diminutive and less expensive devices having sensing, transmitting and processing potentials. Each and Every sensor network node has generally quite a few components such as: a radio transceiver with an inner antenna or a connection to an outer antenna, a microcontroller, an electronic circuit to interface the sensors and energy resource, usually a battery or an implanted form of energy harvesting. Wireless and Tele-communication technology has seen a booming augmentation in this decade. Due to this terrific advancements and user specific requirements, a variety of classes of communication networks have come forward such as Ad hoc Networks, Cellular networks and Sensor Networks. The Cellular Networks depends upon the infrastructure of a network. Ad hoc networks work on multi hop radio relaying because the nodes are energetically and haphazardly located. Ad hoc networks are those which are independent of network's infrastructure [1-3]. In case of sensor networks, nodes compute the circumstances in the atmosphere surrounding them. These measurements are then converted into signals that can be processed to get information regarding the characteristics about the event. The congregated information is then sent to a unique node, called sink node or Base Station (BS). Subsequently, the sink node then passes the data to the user by means of satellite, internet or via gateway. By uniting the advantages of wireless networks with some computational abilities, WSNs have an endless set of potential applications in military and civilian functions together with robotic and land-mine detection. Some of the other applications like goal tracking, battlefield observation, ecological supervising, disaster monitoring, wildfire exposure, industry, safety, cultivation and to monitor natural phenomena etc. [1] Energy efficiency and sensing exposure are important metrics to extend the lifetime and to lessen the consumption of energy by wireless sensor networks. Many protocols have been developed to deal with these concerns amongst which the clustering is considered to be a reasonable method to minimize the consumed energy [4]. A handful of clustering protocols is engaged in the sensing treatment metric. This work suggests a universal configuration that concentrates on both metrics for clustering algorithms in WSN. The energy constraint in WSNs is a major confront to the growth of several potential applications. Numerous research projects inspected such constraints. Hierarchal routing and its protocols are considered one of the most significant routing protocols that are intended to diminish the energy utilization in WSNs [5-7]. Nonetheless, most of such protocols focused on dropping the energy expenditure with no regard to the sensing coverage attain by the network. The main goal of the method is to observe a region to accumulate and pass the information to a sink node or set of sink nodes called as BS [1,3]. In Direct hop communication, each and energy node sends the data to BS directly without the making of connections with other nodes. On the other hand, nodes need to be in contact with neighbor nodes in order to transfer the messages, data or information to BS. WSNs also send the information on the basis of periodicity of data 
transmission which is categorized as:
a) Time-Driven Network
b) Event-Driven Network
c) Query-Driven Network In Timedriven network, every node transmits messages at standard periods, whereas in an Event-driven mode, node sends message only when it sense a phenomenon [9]. The third class is the Query-driven approach in which the sensors send data only when it receives an enquiry from the BS. There are also hybrid networks that fuse together the preceding three models.

\section{Energy Efficient Coverage Issue}

The major difficulty in WSNs is the EEC which occurs primarily in unstructured WSN. For the accomplishment of WSNs, it becomes an important issue to eliminate this problem. As, sensors in the majority of WSNs work with batteries, it is generally not possible to recharge or restore the batteries when they get exhausted. Many methods have been suggested to crack the EEC problem to save energy and extend a network lifetime. Along with these techniques, the device's movement scheduling processes, which depends on the compilation of set of a smallest lively sensor nodes to be wide aware to maintain the coverage of noticeable region, has been revealed to be helpful. Various scheduling algorithms like Greedy Algorithm, Particle Swarm Optimization Algorithm (PSO) and conventional Ant Colony Optimization Algorithms (ACO) have been put forwarded to solve the EEC issue of WSNs. The EEC issue has been transformed into a binary integer programming in greedy algorithm. A basic substitute of PSO algorithm works by having inhabitants called a swarm of contender explanations called particles where the actions of the particles are directed by their own best place in the inspected space as well as the entire swarm's most outstanding accepted site [10].

The conventional ACO is completely based on the swarm intelligence, which states that the complete joint measures come into view from the behaviors of a variety of agents, like ants. The performance of the ACO algorithms is determined by its procedure of initialization of pheromone field. On the other hand, in ACB-SA, a superior method named as Probability Sensor Detection (PSD) model is put forward rather than Boolean Sensor Detection model (BSD) which was used in conventional ACO. BSD is insufficient to find out the accurate results regarding an event because it only depicts the results as success or failure. Therefore, in order to sustain more accuracy, PSD model is being used [10]. The advantage of PSD is that it can easily get used with heterogeneous sensor sets which ensure strong network connectivity than homogeneous sensors. Contrasting convention ACO algorithms, the ACB-SA has also applied to a heterogeneous sensor set which is more sensible and proficient than that of homogeneous sensor set because of their capability to carry out variety of tasks due to diverse set of sensors i.e. heat, light, temperature and humidity sensors etc. The heterogeneous sensor set is also more connection oriented in between WSN and a regional system as discussed above. In addition to this, the two user parameters $\alpha$ and $\beta$ have been removed and there is no need to reflect on the values of these parameters [10].

\section{Low Energy Adaptive Clustering Hierarchy}

Low Energy Adaptive Clustering Hierarchy (LEACH) is a Medium Access Control (MAC) protocol which works on the concept of the formation of CHs within a cluster [11]. Each node in LEACH protocol communicates with another node by the use of unique Code Division Multiple Access (CDMA) technique. This helps in reducing the interference among the nodes belonging to the same cluster. The transmission of data by various nodes is based on the theory of Time Division Multiple Access (TDMA) technique, where each node is assigned with a different schedule $[11,12]$.

LEACH comes under the implementation of hierarchical routing in which Cluster Heads (CHs) are formed within a cluster in order to transfer the data to another $\mathrm{CH}$. Hierarchical or cluster-based routing, was originally proposed in wire line networks having various advantages associated to scalability and affectivity in the communication. Also, the concept of hierarchical routing also helps to enhance the energy-efficient routing in WSNs [11]. The routing in this technique is based on the formation of two tiers of nodes. The nodes with higher energy are implanted to process and send the information whereas the nodes having lower energy are used to achieve the proximity of the target. Hierarchical routing is an effective way to lower down the energy consumption inside a cluster. The collected data after sensing is then fused to compressed data in order to decrease the quantity of messages to be transmitted to BS. Hierarchical routing is principally works upon twolayer routing, where one layer is used for the selection of $\mathrm{CHs}$ and the other one is for routing [13-15]. LEACH appoints a few numbers of nodes as $\mathrm{CHs}$ and put their role in rotation for the even distribution of energy load among the other sensors in the network. The purpose of $\mathrm{CHs}$ is to receive the data from the respective nodes within a cluster and before the transference of this data; the $\mathrm{CHs}$ compress this data for the formation of aggregated form [16]. After compression, the compressed data in the form of aggregated packet is then sent to the BS. By doing this, the load on $\mathrm{CHs}$ is reduces which reduces the consumption of energy [17, 18]. The function of LEACH is composed of two phases, the setup phase and the other is steady state phase. In setup phase, the clusters are formed and selection of CHs takes place. In steady state phase, the nodes start the sensing 
and transference of actual data to BS. The interval of steady state phase is set longer to minimize overhead formation $[11,20]$.

\section{Proposed Methodology}

Energy Efficiency is one of the most considerable concerns in WSNs on which the lifetime of a network can be determined. WSNs are integrated with a small or restricted battery source which is very hard of impossible to recharge after the drainage. Hence, many energy efficient techniques are proposed to deal with this vital issue. Many energy efficient routing protocols and algorithms have been put forward to minimize this issue. But, there are some of the drawbacks faced in all the methods applied. LEACH protocol is one of an efficient protocol used to control this issue but because of the deployment of homogeneous nodes, the protocol is not as efficient as required. Hence, in this paper an improved variant of LEACH protocol is proposed which works on the concept of heterogeneity of nodes so that all the nodes can carry out diverse set of tasks. This variant of LEACH protocol is taken as Improved LEACH. The proposed heterogeneity lies in the term of energy allocated to the nodes. The node with higher energy among other nodes in the cluster becomes the $\mathrm{CH}$ in the proposed variant of LEACH protocol. By this reason, the $\mathrm{CH}$ node can cover or make contact with the far away nodes in order to transfer the data to the larger distances. The overhead also get minimized by the homogeneity in the energy level of the nodes. The comparative analysis of both of the protocols is discussed in the section of results and discussion. The flow of work is discussed in seven stages which are discussed as follows.

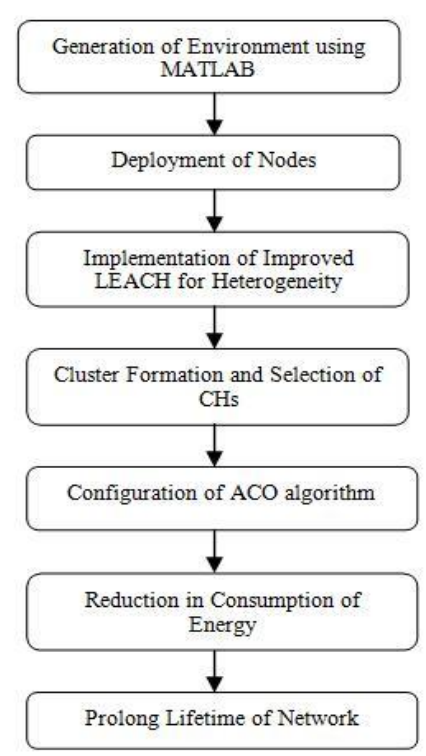

Figure1. Flowchart of Proposed Work

\section{Simulation Results And Discussion}

In this section, simulated results have been carried out which ensures the better performance of ACBSA rather than ACO by using MATLAB 7.11. All simulations have been taken out on a system having Intel Core i3-2330M CPU operation at $2.20 \mathrm{GHz}$ and $4 \mathrm{~GB}$ of RAM. The three scenarios are put into practice with the total number of sensors varying from 100 to 200 and having a difference of 50 from Scenario 1 to Scenario 3. The Point of Interests (PoIs) has also been assigned to every scenario also. The three scenarios and PoIs have been shown in Table 1 .

Table-1.Scenarios and PoIs

\begin{tabular}{c|cc}
\hline Scenario & No. of Sensors & POIs \\
\hline Scenario 1 & 50 & 10 \\
Scenario 2 & 100 & 10 \\
Scenario 3 & 150 & 10 \\
\hline
\end{tabular}

The two simulations have been taken out to confirm the better performance of the ACB-SA as compare to conventional ACO. In the first simulation, the network lifetime and performance is being calculated. The second simulation has been run by means of the ACB-SA in real number space with a heterogeneous sensor set. The position values of the sensors and the PoIs were arbitrarily chosen as real numbers within the range of the monitored region. The network lifetime comparison of two algorithms ACO and ACB-SA is shown in the Table-2. 
Table-2.Network Lifetime Comparison

\begin{tabular}{c|cc}
\hline Scenario & $A C O$ & $A C B-S A$ \\
\hline Scenario 1 & 41.2000 & 48.2000 \\
Scenario 2 & 49.1000 & 54.1000 \\
Scenario 3 & 92.2300 & 103.3000 \\
\hline
\end{tabular}

The Table-2 shows that ACB-SA has better results when it get modeled with probabilistic sensor detection and when it get applied to solve the EEC problem in WSNs. In this case, the major benefit of the system is that it can utilize information collected from real devices without converting them to integers. All sensors in the WSN follow the probabilistic sensor detection model mechanism.

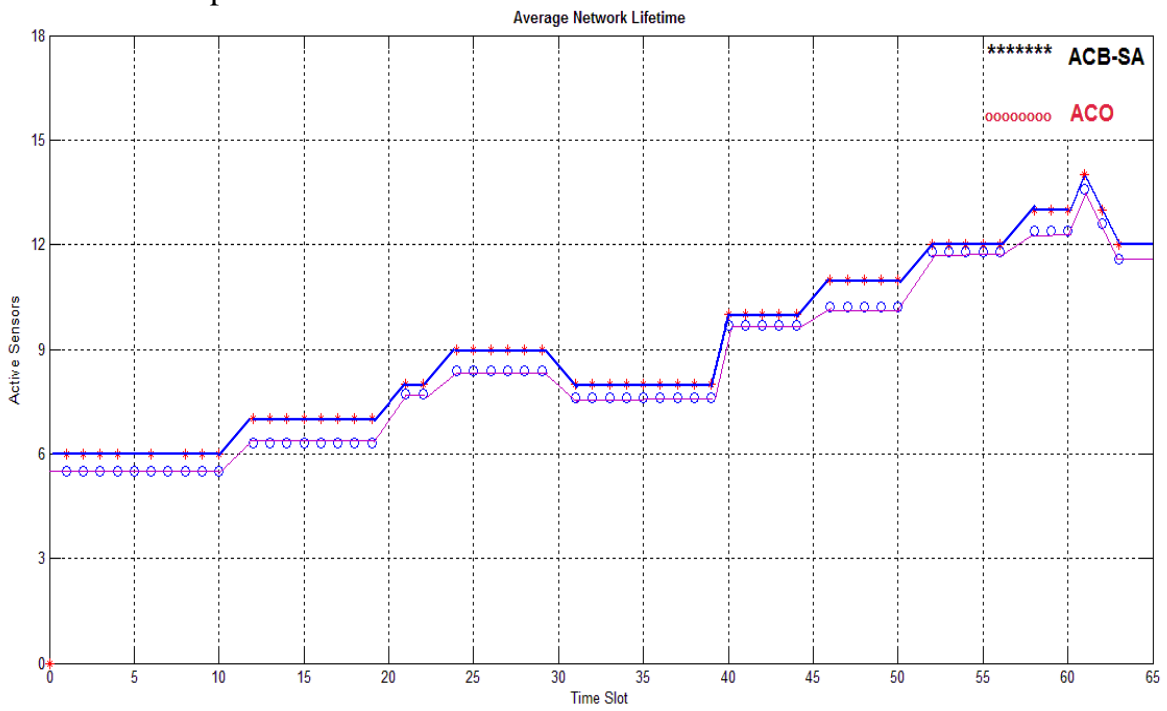

Figure 2. Average Network Lifetime

\section{Conclusion}

In this paper, we have proposed an algorithm which is optimized to solve the EEC issue. The ACB-SA shows innovative uniqueness and has better affectivity than conventional ACO algorithms. The traditional scheduling algorithms which use the ACO algorithm are not optimized to solve the EEC issue. Though, the performance of the ACO algorithm is resolute from its skill to initialize the pheromone field and construction graph which reveal the characteristics of the problem. Additionally, the ACB-SA, distinctly conventional ACO algorithms, does not reflect on what values are advantageous for the user parameters $\alpha$ and $\beta$. The result of the first simulation validates the usefulness of the ACB-SA over other algorithms. The EEC problem is solved by using the ACB-SA via more realistic approach. To accomplish this, a heterogeneous sensor set is used in the WSN, which was prepared by the arbitrary selection of parameters for the probabilistic sensor detection model. We also put forward a solution to EEC problem beneath real number space. The results of the simulations reveal that the ACB-SA can solve the EEC issue through a more practical style.

\section{References}

[1] Akyildiz IF, Sankarabramaniam Y, Cayirci E. A survey on sensor networks. IEEE Communications Magazine. vol.40; 2002, p. $102-114$.

[2] Choi S-H, Kim B-K, Park J, Kang C-H, Eom D-S. An implementation of wireless sensor network. IEEE Transactions on Consumer Electronics. 2004; 50(1):236-244.

[3] Callawa EH. Wireless Sensor Networks: Architectures and Protocols. Boca Raton, FL: Auerbach Publications. 2003.

[4] Sohrabi K, Pottie J. Protocols for self-organization of a wireless sensor network. IEEE Personal Communications. 2000; 7(5):1627.

[5] Awad F, Taqieddin E, Seyam A. Energy-Efficient and Coverage-Aware Clustering in Wireless Sensor Networks. Wireless Engineering and Technology. vol.3; 2012, p. 142-151.

[6] Wang ZM, Basagni S, Melachrinoudis E, Petrioli C. Exploiting Sink Mobility for Maximizing Sensor Networks Lifetime. Proceedings of the 38th Hawaii International Conference on System Sciences. IEEE Computer Society. 2005, p. 1-9.

[7] Jamal N, Karaki Al, Kamal AE. Routing Techniques in Wireless Sensor Networks: A Survey. ICUBE initiative of Iowa State University, Ames, IA 50011. 2004, p. 1-36.

[8] Sun Y, Liu H, M.S. Kim M.S. Energy-Efficient Routing Protocol in Event-Driven Wireless Sensor Networks. IEEE International Conference on Communication Workshops. 2010, p. 1-5.

[9] Elizabeth M, Belding R, Charles EP. Evolution and future directions of the ad hoc on-demand distance-vector routing protocol. Ad Hoc Network. vol.1; 2003, p. 125-150.

[10] Lee JW, Lee JJ. Ant-Colony-Based Scheduling Algorithm for Energy-Efficient Coverage of WSN. IEEE Sensors Journal. 2012; $12(1)$, p. 3036-3046. 
[11] Jamal N, Karaki Al, Kamal AE. Routing Techniques in Wireless Sensor Networks: A Survey. Wireless Communication, IEEE. 2004; 11(6), p. 6-28.

[12] Vidhyapriya R, Vanathi, PT. Energy aware routing for wireless sensor networks. Proceedings of International Conference on Signal Processing, Communications and Networking. 2007; p.545-550.

[13] Jain, Kaushik P, Singhai J. Energy Efficient Maximum Lifetime Routing For Wireless Sensor Network. International Journal of Advanced Smart Sensor Network Systems. 2012; 2(1).

[14] Singh BP, Arora A, Kumar A. Security in Wireless Sensor Networks. Journal of Advances in Physics. 2010; 1(3), p. 1-4.

[15] Singh SK, Singh MP, Singh DK. Routing Protocols in Wireless Sensor Networks - A Survey. International Journal of Soft Computing and Engineering. 2011; 72(8), p. 35-39.

[16] Deepa H, Das AK. A Study on Routing Protocols in Wireless Sensor Network. International Journal of Computer Applications. $2013 ; 72(8)$, p. 35-39.

[17] Geetha V, kallapur PV, Tellajeera S. Clustering in Wireless Sensor Networks: Performance Comparison of LEACH \& LEACH-C Protocols Using NS2. 2nd International Conference on Computer, Communication, Control and Information Technology (C3IT2012) ELSEVIER. 2012; 4, p. 163-170.

[18] Trivedi M, Singh JK. Performance Analysis with Lifetime Optimization of LEACH Routing Protocol for Wireless Sensor Network. International Journal of Engineering Trends and Technology.2014; 8(5), p. 223-226.

\section{Authors' Biography}

Nishant Juneja received his Graduation in B.Sc. in the year 2000 from R.S.D. College, Ferozepur and Post

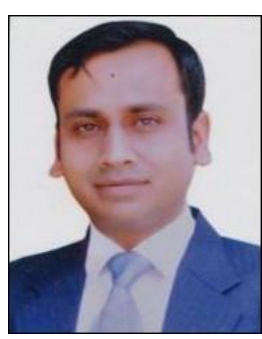
Graduation in Mathematics from Guru Nanak Dev University (campus), Amritsar in 2002. He is currently pursuing Ph.D. in Mathematical Biology from I.K. Gujral Punjab Technical University, Kapurthala. He is serving as Head and Assistant Professor in P.G. Department of Mathematics at Dev Samaj College for Women, Ferozepur. He has published 8 research papers in National as well as International Journals and Conferences. He has work experience of more than 12 years.

Barinder Paul Singh received Bachelor of Technology and Masters of Technology Degrees from Shaheed

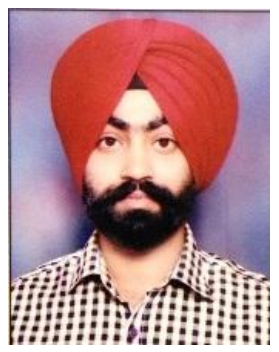
Bhagat Singh State Technical Campus, Ferozepur, India, in 2010 and 2014 respectively in Electronics and Communication Engineering. He is currently working as an Assistant Professor in Department of Electronics and Communication Engineering at Ferozepur Group of Colleges, Ferozeshah. His areas of interest include Wireless Sensor Networks, Ad-hoc Networks, Cellular Communication and Fuzzy Logic. He has 12 publications in international journals and conferences.

\section{IOSR Journal of Electrical and Electronics Engineering (IOSR-JEEE) is UGC approved Journa with Sl. No. 4198, Journal no. 45125.}

